\title{
Measuring Neutrino Masses and Dark Energy
}

\author{
Huitzu Tu \\ UC Irvine
}

June 7, 2007

Dark Side of the Universe, Minnesota, June 5-10 2007

In collaboration with: Steen Hannestad, Yvonne Wong,

Julien Lesgourgues, Laurence Perotto,

Ariel Goobar, Edvard Mörtsell 


\section{Motivation}

Era of precision cosmology: CMB, LSS, SNla, ...

- Neutrino masses ? (0.056 (0.095) $\mathrm{eV}<\sum m_{\nu}<0.2-1.7 \mathrm{eV}, \mathrm{vs} \sim 6 \mathrm{eV}$ from lab)

- Dark energy ? $\left(w \sim-1, \Omega_{X} \sim 0.7, w(z)\right.$ ?)

$\Rightarrow$ Breaking the $\left(\sum m_{\nu}, w\right)$ degeneracy

- Baryon acoustic oscillations detected by SDSS

- Weak lensing (tomography): CFHTLS, SNAP, LSST, ...

- Other observations 


\section{Neutrino Mass Effects}

- Present matter density:

$$
\Omega_{\nu} h^{2} \simeq \frac{\sum m_{\nu}}{93.2 \mathrm{eV}}
$$

- Slow down fluctuation growth:

$$
\Delta P_{\text {lin }}(k) / P_{\text {lin }}(k) \sim-8 \Omega_{\nu} / \Omega_{m} \quad \text { at } k \gg k_{\mathrm{nr}}
$$

[Hu,Eisenstein, Tegmark '97]

- Late time infall into CDM halos:

$$
\rho_{\text {halo }}(r, M, z) \rightarrow \Delta P_{\mathrm{nl}}(k) / P_{\mathrm{nl}}(k) \sim 1 \% \text { at } k \gtrsim 1 h \mathrm{Mpc}^{-1}
$$




\section{Neutrino Mass Effects (II)}
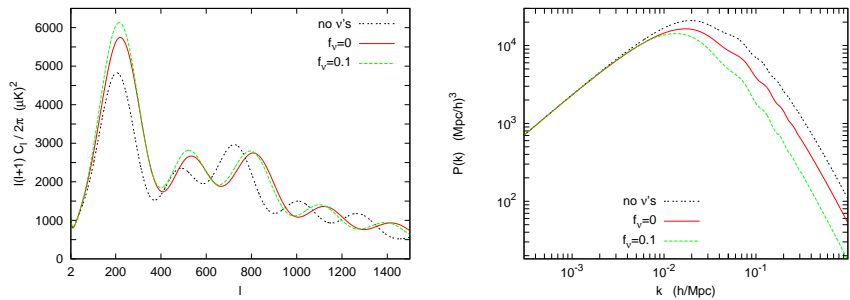

'06]

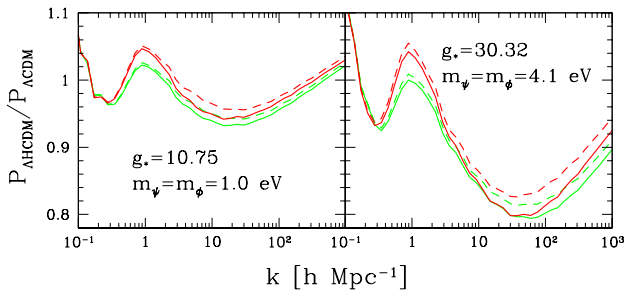

[Hannestad,HT, Wong '05] 


\section{Dark Energy Effects}

- Redshift-distance relation:

$$
H^{2}(z)=H_{0}^{2}\left[\Omega_{m}(1+z)^{3}+\Omega_{X} \exp \left[3 \int_{0}^{z} d z^{\prime} \frac{1+w\left(z^{\prime}\right)}{1+z^{\prime}}\right]\right]
$$

- Structure growth suppression:

$$
\begin{aligned}
& 2 \frac{d^{2} g}{d \ln a^{2}}+\left[5-3 w(a) \Omega_{X}(a)\right] \frac{d g}{d \ln a}+3[1-w(a)] \Omega_{X}(a) g(a)=0 \\
& \text { for the growth function } D(a)=a g(a)
\end{aligned}
$$

Degeneracy with $\sum m_{\nu}$ : due to $\left(w_{X}, \Omega_{X}\right)$ and $\left(\sum m_{\nu}, \Omega_{m}=1-\Omega_{X}\right)$

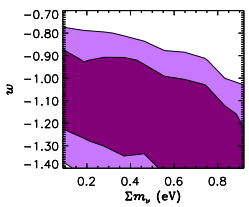

- Baryon Acoustic Oscillations

- Weak gravitational lensing

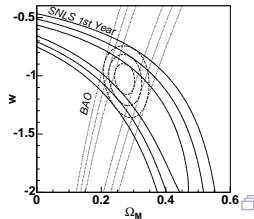




\section{Baryon Acoustic Oscillations (BAO)}

Oscillations in the photon-baryon fluid before recombination [Peeble, Yu $' 70 ; \ldots]$

- Sound horizon at recombination

$$
r_{s}\left(\eta_{\mathrm{rec}}\right) \approx 147 \operatorname{Mpc}\left(\Omega_{m} h^{2} / 0.13\right)^{-0.25}\left(\Omega_{b} h^{2} / 0.024\right)^{-0.08}
$$

- "Standard ruler":

$$
r_{\|}=\Delta z / H(z), r_{\perp}=(1+z) D_{A}(z) \Delta \theta
$$
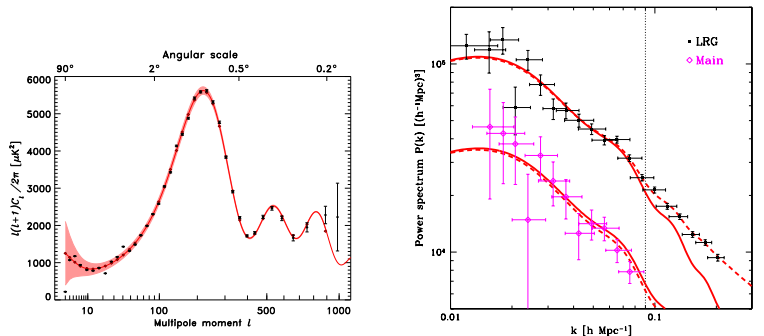


\section{BAO (II): SDSS Detection in Galaxy Correlation Function}

$\xi(\vec{r}) \equiv\langle\delta(\vec{x}) \delta(\vec{x}+\vec{r})\rangle \Rightarrow \xi(r)=\frac{1}{(2 \pi)^{3}} \int d k P(k) \frac{\sin k r}{k r} 4 \pi k^{2}$

$\Rightarrow$ SDSS constraints on dark energy:

\begin{tabular}{lll} 
parameter & WMAP + Main & WMAP + LRG \\
\hline $\mathrm{w}$ (constant) & $-0.92 \pm 0.30$ & $-0.80 \pm 0.18$
\end{tabular}

$\Rightarrow$ With massive neutrinos we found at $z=0.35$

[Goobar,Hannestad,Mörtsell,HT '06]

$$
A \equiv D_{V}(z) \frac{\sqrt{\Omega_{m} H_{0}^{2}}}{c z}=0.469\left(\frac{n}{0.98}\right)^{-0.35}\left(1+0.94 \Omega_{\nu} / \Omega_{m}\right) \pm 0.017
$$

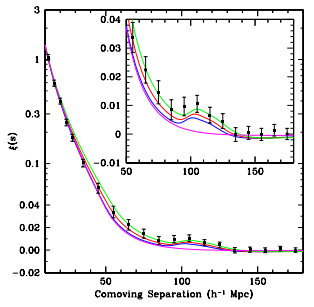




\section{Our Current Bounds on $\sum m_{\nu}: 2$ models}

11 parameters: $\left(\Omega_{m}, h, \Omega_{b} h^{2}, N_{\nu}, w_{x}, n_{s}, \alpha_{s}, \tau, Q, b, \sum m_{\nu}\right)$

Data

$\frac{\sum m_{\nu}(95 \% \text { C.L. })}{1.72 \mathrm{eV}}$

2. $\mathrm{CMB}, \mathrm{LSS}, \mathrm{SNla}, \mathrm{BAO}$

$0.62 \mathrm{eV}$

3. CMB, LSS, SNla, Ly- $\alpha$

$0.83 \mathrm{eV}$

4. CMB, LSS, SNIa, BAO, Ly- $\alpha$

$0.49 \mathrm{eV}$

8 parameters: $\left(\ldots, w_{X}=-1, \alpha_{s}=0, N_{\nu}=3\right)$

Data

$\frac{\sum m_{\nu}(95 \% \text { C.L. })}{0.70 \mathrm{eV}}$

1: CMB, LSS, SNla

2. $\mathrm{CMB}, \mathrm{LSS}, \mathrm{SNla}, \mathrm{BAO}$

$0.48 \mathrm{eV}$

3. CMB, LSS, SNla, Ly- $\alpha$

$0.35 \mathrm{eV}$

4. CMB, LSS, SNIa, BAO, Ly- $\alpha$

$0.27 \mathrm{eV}$ 


\section{Future BAO Surveys}

Statistical errors $\frac{\sigma_{P}}{P}(k) \approx 2 \pi \sqrt{\frac{1}{V_{\text {survey }} k^{2} \Delta k}}\left(1+\frac{1}{n P(k)}\right)$

- Large volume $\left(\sim 1 \mathrm{Gpc}^{3}\right)$

- Aim at high redshifts $\rightarrow$ still in linear regime

- Need spectroscopic redshifts (otherwise $\approx 20$ larger area)

- With redshift slicing (5-7 slices, $1<z<3) \rightarrow w(z)$

WFMOS, HETDEX, JEDI, LSST, VADER (?)...

- Constraints on dark energy: $\sigma\left(w_{0}\right) \sim 10 \%$ and $\sigma\left(w_{1}\right) \sim 20 \%$ [Glazebrook, Blake '05; Wang '06;...]

- Constraints on neutrino masses: a full MCMC to be done

[Hannestad, Lesgourgues, Perotto, HT, Wong, in preparation]

- Model/scenario comparison: with $H(z)$ and $D_{A}(z)$ data [Goobar, Hannestad, Mörtsell,HT] 


\section{Future BAO Surveys (II)}

- Dependence on fiducial model

- Sensitivity comparison to weak lensing
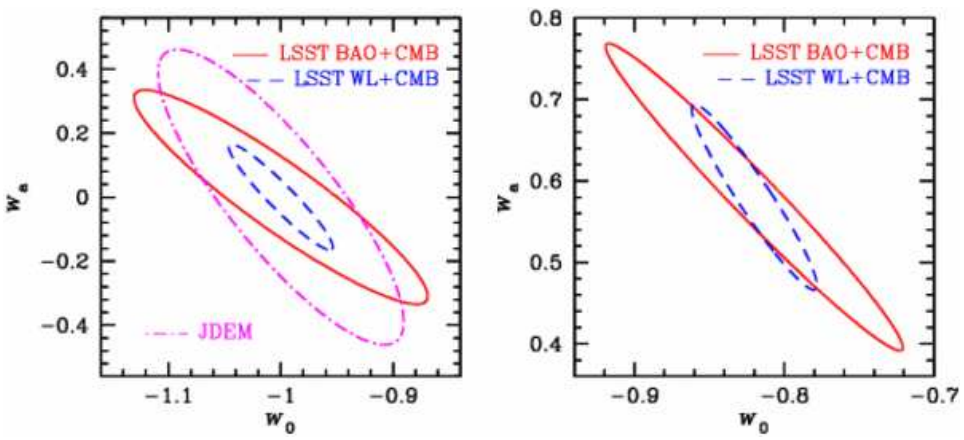

[LSST Collaboration, http://www.lsst.org] 


\section{Gravitational Weak Lensing (Tomography)}

Convergence power spetrum

$$
C_{\ell}^{i j}=\frac{9}{16} H_{0}^{4} \Omega_{m}^{2} \int_{0}^{\chi_{h}} d \chi \frac{g_{i}(\chi) g_{j}(\chi)}{a^{2} \chi^{2}} P\left(\frac{\ell}{\chi}, z\right)
$$

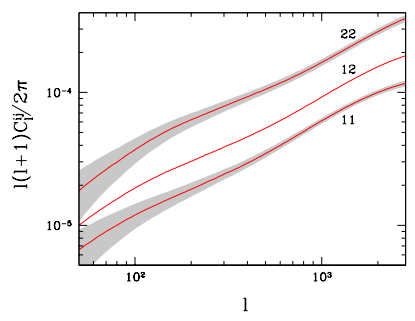

Huitzu Tu UC Irvine

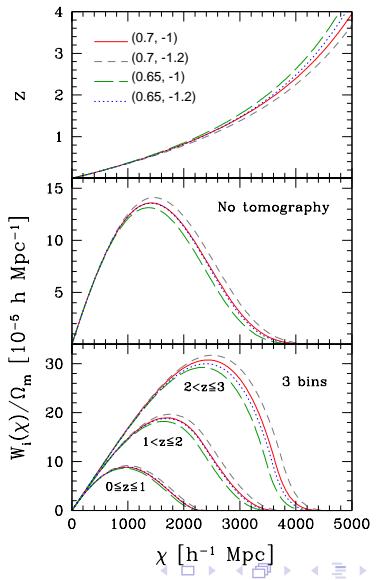

Measuring Neutrino Masses and Dark Energy 


\section{WL Systematics}

- Photometric redshift uncertainties $\sim 0.05$

$$
n_{i}(z)=\int_{z_{\mathrm{ph}}^{i}}^{z_{\mathrm{ph}}^{i+1}} d z_{\mathrm{ph}} n_{\mathrm{gal}}(z) p\left(z_{\mathrm{ph}} \mid z\right) \Rightarrow \sigma_{z}, z_{\text {bias }}
$$

- Shear calibration

- Multiplicative errors $\sim 1-2 \%$

$$
\hat{C}_{\ell}^{i j}=C_{\ell}^{i j} \times\left(1+f_{i}+f_{j}\right)
$$

- Additive errors $~ 10^{-4}$

$$
\hat{C}_{\ell}^{i j}=C_{\ell}^{i j}+C_{\ell}^{\text {add }}
$$

- Nonlinear corrections to matter power spectrum $\sim 5-10 \%$ "halo model" $\Rightarrow P_{\mathrm{nl}}(k)$ 


\section{Our Analysis: Parameters and Surveys}

- WL tomography with 1,3,5,8 bins, $0<z<3$

- Fiducial cosmological model (11 parameters):

$$
\begin{aligned}
& \left(w_{0}=-1, w_{a}=0, \sum m_{\nu}=0.07 \mathrm{eV}, N_{\mathrm{eff}}=3,\right. \\
& \Omega_{c} h^{2}=0.1225, \Omega_{\mathrm{DE}}=0.7, \Omega_{b} h^{2}=0.0245, n_{s}=1, \\
& \left.\alpha_{s}=0, \tau=0.05, \sigma_{8}=0.9\right)
\end{aligned}
$$

- Systematics: priors on $\sigma_{z}=0.05$ and $z_{\text {bias }}=0, f_{i}=0$

- Generic WL survey parameters:

\begin{tabular}{lcccc} 
& $f_{\text {sky }}$ & $z_{0}$ & $\gamma_{\text {rms }}$ & $\bar{n}_{\text {gal }}\left(\operatorname{arcmin}^{-2}\right)$ \\
\hline Wide, LSST-like & 0.7 & 1.0 & 0.4 & 30 \\
Deep, SNAP-like & 0.01 & 1.5 & 0.25 & 100
\end{tabular}




\section{Error Forecast for $\sum m_{\nu}$ and $w$}

\begin{tabular}{llc} 
Model & Cosmological probes & $\sigma\left(\sum m_{\nu}\right)$ \\
\hline 11 parameters & Planck only & $0.48 \mathrm{eV}$ \\
11 parameters & Planck + Wide- 1 & $0.15 \mathrm{eV}$ \\
11 parameters & Planck + Wide- 5 & $0.043 \mathrm{eV}$ \\
7 parameters & Planck + Wide- 1 & $0.082 \mathrm{eV}$ \\
7 parameters & Planck + Wide- 5 & $0.037 \mathrm{eV}$
\end{tabular}

WL tomography (wide) + Planck

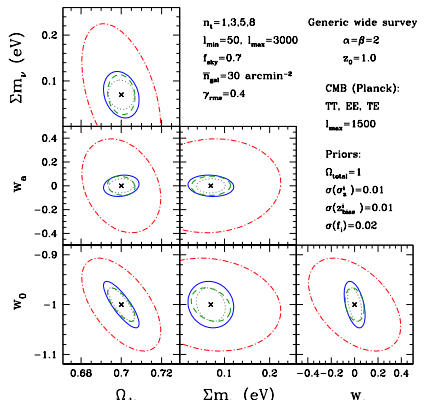

Huitzu Tu UC Irvine 


\section{Summary}

Breaking $\left(\sum m_{\nu}, w\right)$ degeneracy

- $\sum m_{\nu} \leq 0.48-0.62$ from WMAP3 + LSS + SNla + BAO

- Future WL tomography constraints: $\sigma\left(\sum m_{\nu}\right)=0.025-0.1 \mathrm{eV}$

- CMB lensing $\sigma\left(\sum m_{\nu}\right) \sim 0.15 \mathrm{eV}$ (Planck)

$\Rightarrow$ Cosmological observations pin down $\sum m_{\nu}$ to $<0.1 \mathrm{eV}$, distinguish between normal hierarchy and inverted hierarchy

- $\sigma\left(w_{0}\right) \sim 10 \%, \sigma\left(w_{a}\right) \sim 20 \%$ from future BAO

- $\sigma\left(w_{0}\right) \sim 3.5 \%, \sigma\left(w_{a}\right) \sim 8 \%$ from future WL with 5 bins 